\title{
Cineventriculographic Analysis of the Ventricular Septal Motion during Stimulation of Various Pacemaker Sites
}

\author{
Kozui Miyazawa, Takao Honna, Takashi Haneda, Kunio \\ Shirato, Toshiyuki Nakajima and Toru AraI \\ The First Department of Internal Medicine, Tohoku University \\ School of Medicine, Sendai 980
}

\begin{abstract}
Mryazawa, K., Honna, T., Haneda, T., Shirato, K., Nakajmua, T. and Akar, T. Cineventriculographic Analysis of the Ventricular Septal Motion during Stimulation of Various Pacemaker Sites. Tohoku J. exp. Med., 1978, 126 (4), 363-369 - By means of cineventriculography, contraction pattern of the ventricular septum was studied during electrical stimulation of the right atrium (RA), outflow tract (RVO) and apex (RVA) of the right ventricle and apex (LVA) and lateral wall (LVL) of the left ventricle. RA pacing produced a monophasic shortening of the septal hemiaxes after initial lengthening for about $40 \mathrm{msec}$ from $R$ wave of ECG. In contrast, ventricular stimulation near the septum (i.e. RVO, RVA and LVA) resulted in a biphasic and decreased contraction of the septal wall, in which an early preejection shortening was followed by a brief lengthening and second shortening. LVL pacing showed a monophasic and increased contraction of the septal wall. The biphasic motion of the septal wall was likely to be related to the abnormal mechanical contraction caused by the abnormal conduction sequence of ventricular depolarization. On the other hand, the lateral wall demonstrated a biphasic and decreased contraction during LVL pacing, and a monophasic and increased contraction during RVO, RVA and LVA pacing. Thus, the biphasic and diminished contraction at the site of electrical stimulation was compensated by the monophasic and forceful contraction of the contralateral wall. It is considered that the ventricular septum plays an important functional role in regulation of cardiac performance.—_- ventricular septum; left ventriculogram; ventricular asynchrony; cineradiography; pacemaker site
\end{abstract}

It has been known that cardiac output and systemic blood pressure during ventricular pacing are lower than those observed during right atrial pacing at the same rate. Our previous study, using two straingauge arches attached to the apical and lateral walls of the left ventricle, demonstrated that the decrease in cardiac performance during ventricular pacing was related to the severity of asynchrony rather than the change in regional myocardial tension (Miyazawa et al. 1977). The interventricular septum appears anatomically to be a part of the left ventricle, but relatively little is known about the functional contribution of the septum to the left ventricle. From this standpoint, the cineventriculographic and cineradiographic studies were designed to analyze the dynamic behavior of the septum together with that of contralateral free wall during atrial and ventricular rhythm

Received for publication, March 3, 1978. 
and to clarify the mechanism by which contraction of the septum contributes to cardiac performance.

\section{Materials and Methods}

Four mongrel dogs weighing 11 to $20 \mathrm{~kg}$ (average weight $14.5 \mathrm{~kg}$ ) were anesthetized with $30 \mathrm{mg} / \mathrm{kg}$ pentobarbital sodium (Nembutal). Bilateral thoracotomy was performed through the fifth intercostal space. The heart was cradled in the open pericardium. Using a respirator (Aika, Model 60) connected to an endotracheal tube, the lung was ventilated with $100 \%$ oxygen at a rate of 25 times per min. A 7 F NIH catheter was introduced from the right femoral artery into the left ventricle. A 7F standard end-hole catheter was placed in the abdominal aorta by way of the left femoral artery for pressure tracing. Lead II of the electrocardiogram and aortic pressure were monitored throughout each experiment. Two radiopaque markers (metal clip) were fixed to the surface of basal and apical regions of the ventricular septum by means of following technique. A marker was

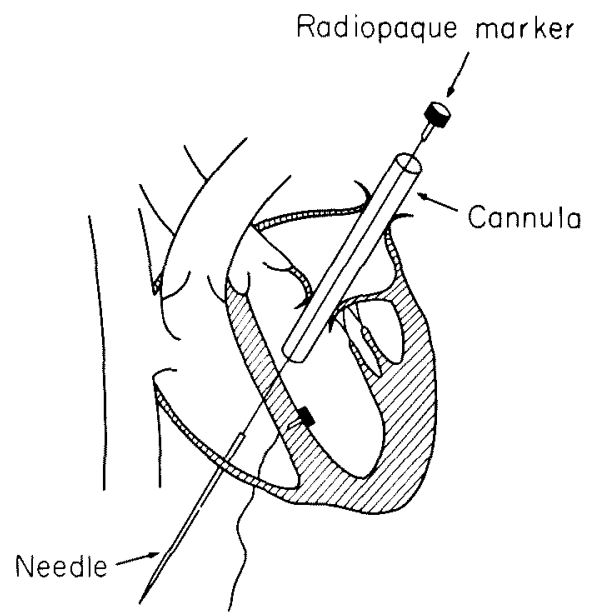

Fig. 1. Schematic illustration of the technique for fixing the radiopaque markers to the ventricular septum.

connected with thread and needle, as shown in Fig. 1. A cannula (length $10 \mathrm{~cm}$, inner diameter $7 \mathrm{~mm}$ ) inserted in the left atrial appendage was passed to the left ventricle across the mitral valve. The needle was introduced into this cannula, pierced through the septum and pulled until the marker was fixed firmly to its surface. Another marker was also placed on the surface of the septum, so that two markers lay on a vertical plane passing through the long axis of the left ventricle. The accuracy of the placement of these markers was later confirmed at autopsy. Bundle branch block was not produced by this procedure. A pair of electrodes was attached to the right atrial appendage (RA), outflow tract (RVO) or apex (RVA) of the right ventricle, or apex (LVA) or lateral wall (LVL) of the left ventricle. Monophasic impulses of 4 to 5 msee duration and 3 to 8 volts intensity were delivered from a stimulator (Nihon Kohden Model SEN 1101). Each dog was secured to an $\mathbf{x}$-ray table in the left anterior oblique position. Thus, the markers outline the profile of the ventricular septum in this position. The sinus node was crushed and electrical stimulation was made at a rate of 150 beats per min. Pacing was first applied to RA and then to RVO, RVA, LVA and LVL. Left ventriculography was performed after a steady state had been attained following transfer of the pacing site. Respiration was interrupted in inspiration before cineventriculography. Ventriculograms were recorded on $16 \mathrm{~mm}$ film at 100 frames per sec utilizing a 9 inch image intensifier system. Contrast medium $(0.8 \mathrm{ml} / \mathrm{kg}$ of $76 \%$ Diatrizoate) was injected at a rate of $17 \mathrm{ml} / \mathrm{sec}$ by a power injector (Contrac $3 \mathrm{E}$, 
Siemens). The signal triggered by $\mathrm{R}$ wave of ECG was recorded on the films to identify the end-diastole. If a premature beat occurred during ventriculography, the beat and the next beat following the premature beat were not analyzed. In order to avoid the hemodynamic effects of the contrast medium, about $15 \mathrm{~min}$ were allowed to elapse between successive angiograms. Using a motion analyzer, the angiographic outline of the left ventricular cavity after the onset of shortening was traced frame by frame. The major axis was determined by the line drawn from the mid point of the aortic valve to the apex of the left ventricle. The image was divided into quarters by three equidistant minor axes perpendicular to the major axis. Each minor axis was sectioned in two parts (medial and lateral hemiaxes). The shortening of the axis was expressed as the percent change of end-diastolic length. Images from successive frames were also superimposed for analysis of the angular change in long axis and the movement of the marker. In these, the code number taped to the image intensifier before filming, spine, ribs and diaphragm were used as the fixed points. The movement of the marker and the distance between two markers were measured in the cineradiograms taken immediately before contrast medium injection.

\section{Results}

Table 1 summarizes the percent shortening of each hemiaxis. Fig. 2 shows the time course of the dimensional changes in septal (medial) hemiaxes during the cardiac cycle. Motion analysis during RA pacing showed a monophasic contraction after initial lengthening for about $40 \mathrm{msec}$ from $R$ wave at the apical and middle segments and $90 \mathrm{msec}$ at the basal segment. Electrical stimulation near the ventricular septum, i.e. RVO, RVA and LVA, caused a biphasic and decreased contraction, showing an early preejection shortening followed by a brief lengthening which was in turn followed by a second shortening. With RVA and LVA pacing, this lengthening attained a peak value during early ejection of less than $100 \mathrm{msec}$ after the onset of mechanical systole, while it was midejection period with RVO pacing. LVL pacing was associated with a monophasic and increased contraction of the septal hemiaxes.

The lateral wall showed a monophasic and increased contraction during RVO, RVA and LVA pacing, while a biphasic and decreased contraction was observed dur-

TABLE 1. Percent shortening of each hemiaxis

\begin{tabular}{lccccc}
\hline & \multicolumn{5}{c}{ Site of electrical stimulation } \\
\cline { 2 - 5 } & RA & RVO & RVA & LVA & LVL \\
\hline Septal hemiaxis & & & & & \\
Basal & $67 \pm 13$ & $40 \pm 10$ & $23 \pm 9$ & $52 \pm 10$ & $64 \pm 18$ \\
Middle & $57 \pm 9$ & $67 \pm 8$ & $47 \pm 10$ & $\mathbf{7 3} \pm 12$ & $87 \pm 23$ \\
$\quad$ Apical & $64 \pm 12$ & $73 \pm 8$ & $44 \pm 9$ & $66 \pm 12$ & $92 \pm 23$ \\
Lateral hemiaxis & & & & & \\
$\quad$ Basal & $26 \pm 2$ & $25 \pm 7$ & $26 \pm 3$ & $\mathbf{2 3 \pm 6}$ & $19 \pm 6$ \\
Middle & $31 \pm 5$ & $26 \pm 6$ & $32 \pm 5$ & $51 \pm 12$ & $21 \pm 5$ \\
Apical & $51 \pm 6$ & $48 \pm 8$ & $47 \pm 16$ & $\mathbf{7 4 \pm 1 0}$ & $47 \pm 14$ \\
\hline
\end{tabular}

Mean \pm s.D., $n=4$.

Abbreviations: RA, right atrium; RVO, outflow tract of right ventricle; RVA, apex of right ventricle; LVA, apex of left ventricle; LVL, lateral wall of left ventricle. 

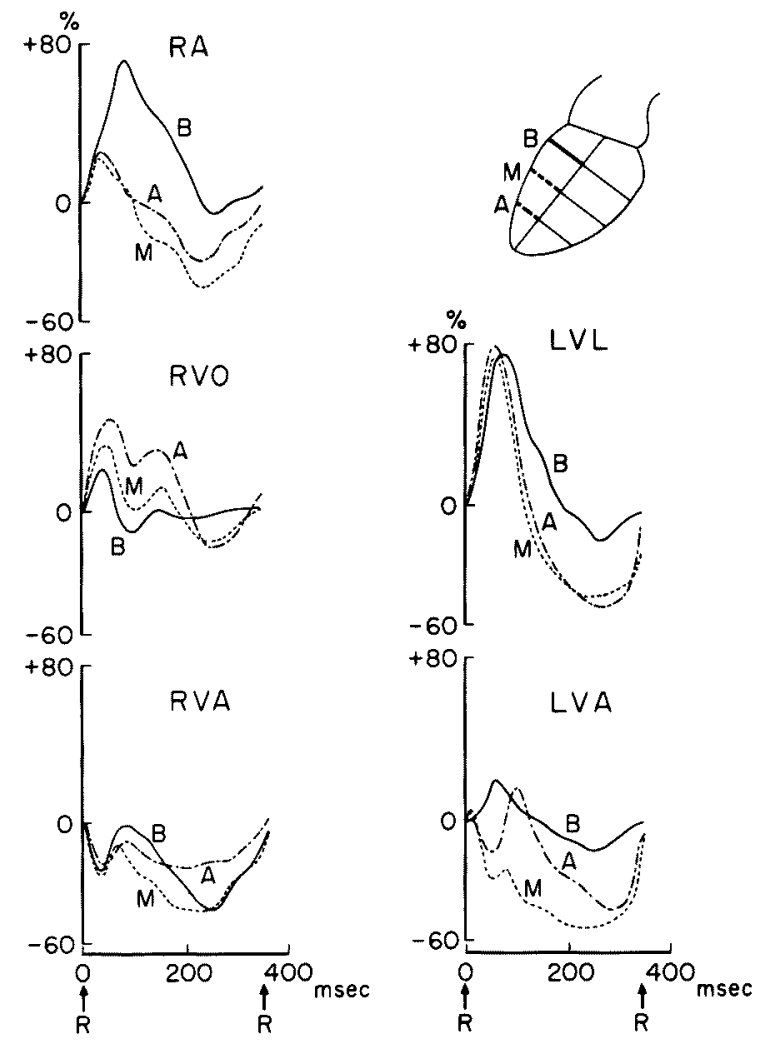

Fig. 2. Time course of percent change in septal hemiaxis during the cardiac cycle. Record obtained from a $\operatorname{dog}(\mathrm{C}-10,12 \mathrm{~kg})$ during electrical stimulation of the right atrium (RA) and various ventricular sites. $B$, basal segment; $M$, middle segment; $A$, apical segment; RVO \& RVA, outflow tract and apex of the right ventricle; LVA \& LVL, apex and lateral wall of the left ventricle; $R, R$ wave of electrocardiogram.

ing LVL pacing (Fig. 3).

With RA pacing, the apical marker moved slightly towards the anterocaudal direction during preejection phase of systole followed by posterior movement during ejection phase and then back to original position, demonstrating a counterclockwise rotation (Fig. 4). RVO, RVA and LVA pacing caused more complicated motion. The marker during LVL pacing was seen to execute a similar counterclockwise rotation to RA pacing, although the amplitude of the movement in anteroposterior direction was somewhat larger.

The percent shortening of the distance between two markers, which represents the dimensional changes in longitudinal direction of the septum, was $24 \pm 4 \%$ for RA pacing, $23 \pm 3 \%$ for RVO, $23 \pm 3 \%$ for RVA, $17 \pm 4 \%$ for LVA and $22 \pm 3 \%$ for LVL, respectively. As shown in Fig. 5, shortening pattern of the distance during systole was monophasic in no relation to the electrical stimulation site.

Angular displacement of the major axis during systole was minimal and the 

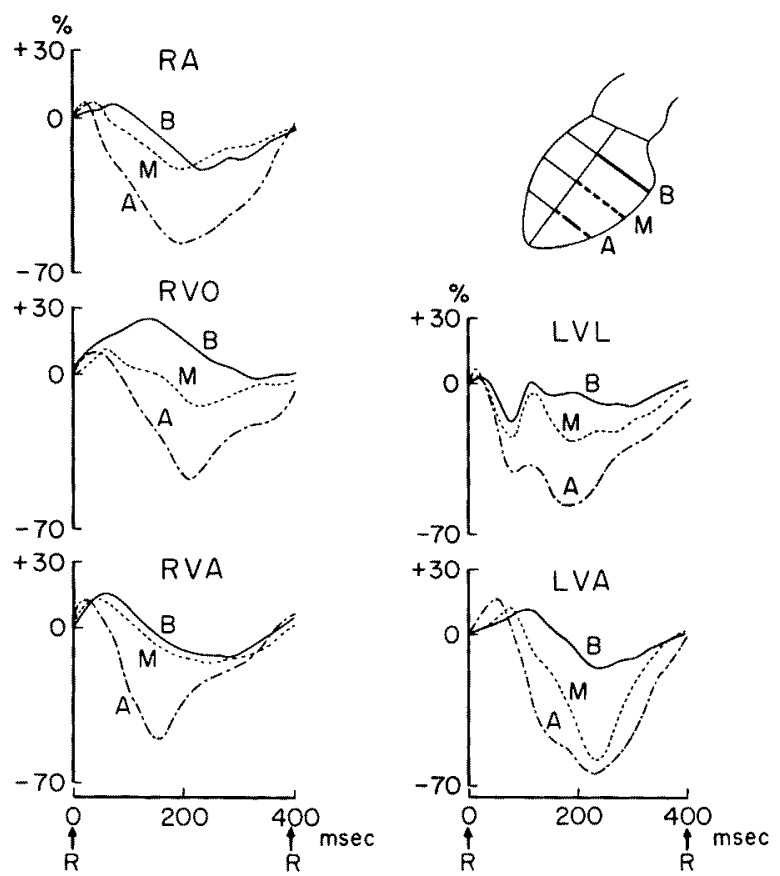

Fig. 3. Time course of percent change in lateral hemiaxis during the cardiac cycle. Record obtained from a $\operatorname{dog}(\mathrm{C}-11,11 \mathrm{~kg})$. Abbreviations are as in Fig. 2.

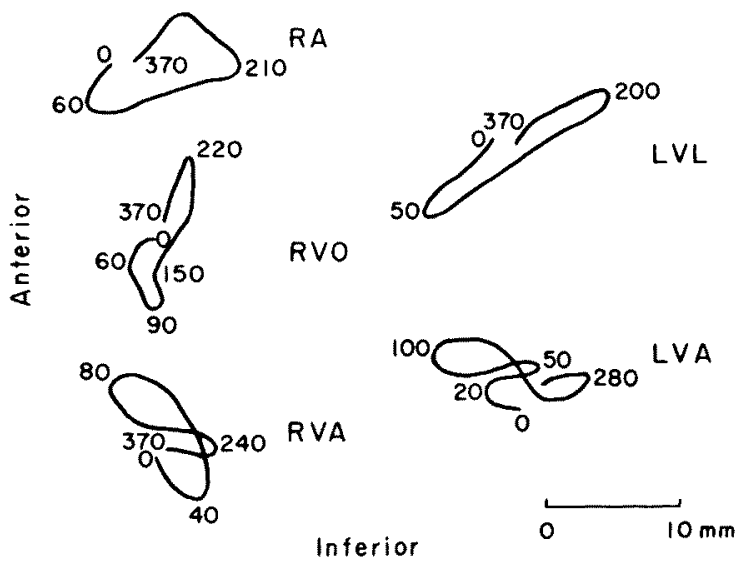

Fig. 4. Motion analysis of the marker attached to the apical region of the septum. Numerals indicate time in msec from $\mathrm{R}$ of ECG. Record obtained from a dog (C-12, $20 \mathrm{~kg}$ ). Abbreviations as in Fig. 2 .

shift was 6.6, 9.0, 8.1, 6.6 and 9.1 degrees, for RA, RVO, RVA, LVA and LVL pacing, respectively. 

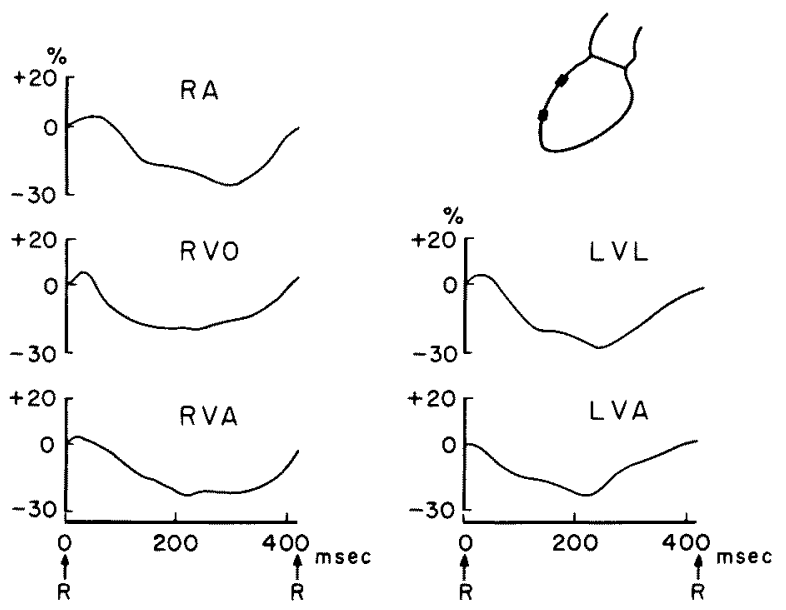

Fig. 5. Tims course of percent change in distance between two markers attached to the septum along the longitudinal direction. Record obtained from a dog $(\mathrm{C}-10,12 \mathrm{~kg})$. Abbreviations as in Fig. 2.

\section{Discussion}

The present study was designed to measure the motion pattern of the interventricular septum in association with contralateral wall. With RA pacing, the septal hemiaxes showed a monophasic contraction of apical segment followed by the delayed shortening of the basal segment beginning approximately $50 \mathrm{msec}$ later. Contraction pattern of the lateral hemiaxes was similar to the septal one. With RVO, RVA and LVA pacing, the septal hemiaxes showed a biphasic contraction pattern, and LVL pacing resulted in a monophasic pattern. On the other hand, the lateral hemiaxes demonstrated a monophasic contraction during ventricular pacing near the septum, and a biphasic contraction during LVL pacing. The reason for early systolic biphasic motion (rocking motion) of the ventricular wall near the stimulation site is not clear, but it may be associated with the abnormal spread of mechanical contraction caused by the abnormal conduction sequence of electrical depolarization. Gomes et al. (1977) reported that using echocardiography, an early rapid preejection motion of posterior septum was uniformly observed during RVO, RVI (inflow tract of the right ventricle) and RVA pacing, and this abnormal motion would be dependent on the sequence of ventricular activation and consequent differential contraction.

It was notable that a monophasic contraction of the septal or lateral hemiaxis during ventricular pacing was associated with an increased contraction of that hemiaxis, while a biphasic contraction was accompanied by a decreased contraction. In estimation of the lengthening and shortening of the hemiaxis, we employed the major axis as a reference axis. However, when large shifts occur about the major axis during cardiac cycle, significant movements of the heart in the chest may be suspected. Present study demonstrated that the angular movement 
in this plane was less than 10 degrees with every pacing site. Rushmer et al. (1953), Harrison et al. (1963), Linch and Rove (1969) and McDonald (1970) also reported that the heart did not move significantly within the chest during contraction, if the diaphragm remained stationary. It is thus presumed that the time course of the dimensional change in septal hemiaxis is similar to the absolute motion of the ventricular wall. In fact, the shortening of apical hemiaxis plotted against time was consistent with the locus of the marker attached to the apical segment of the septum.

The relative contribution of the shortening of longitudinal direction of the septum to left ventricular contraction was also studied by measuring the distance of two markers. The percent shortening of the distance was smaller than that of circumferential direction in no relation to the site of electrical stimulation. Accordingly, pressure development and blood ejection were thought to be more dependent upon the circumferential contraction than the shortening of longitudinal length.

As reported previously, cardiac performance was lower during ventricular pacing when compared with RA pacing, and there were no significant differences by changing the ventricular pacing site (Miyazawa et al. 1976, 1977, 1978). The present study demonstrated that the biphasic and diminished contraction of the septal or lateral wall was related to the monophasic and increased contraction of the lateral or septal wall, respectively. This may signify that ventricular asynchrony maximal at the site of stimulation is compensated by the forceful contraction of the contralateral wall. It is therefore considered that the ventricular septum have demonstrated characteristic contractile behavior which not only acts as a fulcrum but also plays an important role in regulation of cardiac performance.

\section{References}

1) Gomes, J.A.C., Damato, A.N., Akhter, M., Dhatt, M.S., Calon, A.H., Reddy, C.P. \& Moran, H.E. (1977) Ventricular septal motion and left ventricular dimensions during abnormal ventricular activation. Amer. J. Cardiol., 39, 641-650.

2) Harrison, D.C., Goldblatt, A. \& Braunwald, E. (1963) Studies on cardiac dimensions in intact, unanesthetized man: Description of techniques and their validation. Circulat. Res., 13, 448-455.

3) Linch, P.R. \& Bove, A.A. (1969) Geometry of the left ventricle as studied by a highspeed cineradiographic technique. Fed. Proc., 28, 1330-1333.

4) McDonald, I.G. (1970) The shape and movements of the human left ventricle during systole. Amer. J. Cardiol., 26, 221-230.

5) Miyazawa, K., Shirato, K., Haneda, T., Honna, T., Arai, T. \& Nakajima, T. (1976) Effects of varying pacemaker sites on left ventricular performance. Tohoku $J$. exp. Med., 120, 301-308.

6) Miyazawa, K., Arai, T., Shirato, K., Haneda, T., Ikeda, S., Honna, T., Nakajima, T. \& Miura, T. (1977) Regional contraction patterns of the left ventricle during ventricular pacing. Tohoku J. exp. Med., 122, 167-174.

7) Miyazawa, K., Honna, T., Haneda, T., Shirato, K., Nakajima, T. \& Arai, T. (1978) Dynamic geometry of the left ventricle during ventricular pacing: Correlation with cardiac pumping action. Tohoku J. exp. Med., 124, 261-266.

8) Rushmer, R.F., Crystal, D.K. \& Wagner, C. (1953) The functional anatomy of ventricular contraction. Circulat. Res., 1, 162-170. 